\title{
Generalizations of integral inequalities for functions whose second derivatives are convex and $m$-convex
}

\author{
M. Emin Özdemir, Alper Ekinci, and Ahmet Ocak \\ Akdemir
}




\title{
GENERALIZATIONS OF INTEGRAL INEQUALITIES FOR FUNCTIONS WHOSE SECOND DERIVATIVES ARE CONVEX AND $m-$ CONVEX
}

\author{
M. EMIN ÖZDEMIR, ALPER EKINCI, AND AHMET OCAK AKDEMIR
}

Received 21 October, 2011

\begin{abstract}
In this paper, we establish some new integral inequalities for convex and $m$-convex functions by using a new kernel. The analysis used in the proofs is fairly elementary and based on the classical inequalities. We also give some comparisons and applications to special means for our results.
\end{abstract}

2000 Mathematics Subject Classification: 26D15

Keywords: convex functions, $m$-convex functions, Simpson's inequality, power mean inequality

\section{INTRODUCTION}

The following definition is well known in the literature: a function $f: I \rightarrow \mathbb{R}, \varnothing \neq$ $I \subseteq \mathbb{R}$, is said to be convex on $I$ if the inequality

$$
f(t x+(1-t) y) \leq t f(x)+(1-t) f(y)
$$

holds for every $x, y \in I$ and $t \in[0,1]$.

Definition 1 (See [10]). A function $f:[0, b] \rightarrow \mathbb{R}$ is said to be $m$-convex, where $m \in[0,1], b>0$, if for every $x, y \in[0, b]$ and $t \in[0,1]$ we have :

$$
f(t x+m(1-t) y) \leq t f(x)+m(1-t) f(y) .
$$

For recent results related to $m$-convex functions we refer the interest of readers to the papers $[2,3,5,7,8,10]$.

Many authors have been studied on integral inequalities. One of the well known of these inequalities -Simpson's inequality- is given as following:

Theorem 1. Let $f:[a, b] \rightarrow \mathbb{R}$ be a four times continuously differentiable mapping on $(a, b)$ and $\left\|f^{(4)}\right\|_{\infty}=\sup _{x \in(a, b)}\left|f^{(4)}(x)\right|<\infty$. Then, the following inequality 
holds:

$$
\left|\frac{1}{3}\left[\frac{f(a)+f(b)}{2}+2 f\left(\frac{a+b}{2}\right)\right]-\frac{1}{b-a} \int_{a}^{b} f(x) d x\right| \leq \frac{1}{2880}\left\|f^{(4)}\right\|_{\infty}(b-a)^{4} .
$$

In [9], Sarkaya et al. proved the following lemma and established some new Simpson type inequalities for convex functions:

Lemma 1. Let $f: I \subset \mathbb{R} \rightarrow \mathbb{R}$ be a twice differentiable mapping on $I^{\circ}$ such that $f^{\prime \prime} \in L_{1}[a, b]$, where $a, b \in I$ with $a<b$, then the following equality holds:

$$
\begin{aligned}
& \frac{1}{6}\left[f(a)+4 f\left(\frac{a+b}{2}\right)+f(b)\right]-\frac{1}{b-a} \int_{a}^{b} f(x) d x \\
= & (b-a)^{2} \int_{0}^{1} k(t) f^{\prime \prime}(t b+(1-t) a) d t
\end{aligned}
$$

where

$$
k(t)=\left\{\begin{array}{lc}
\frac{t}{2}\left(\frac{1}{3}-t\right), & t \in\left[0, \frac{1}{2}\right) \\
(1-t)\left(\frac{t}{2}-\frac{1}{3}\right), & t \in\left[\frac{1}{2}, 1\right]
\end{array} .\right.
$$

Theorem 2 (See [9]). Let $f: I \subset \mathbb{R} \rightarrow \mathbb{R}$ be a twice differentiable mapping on $I^{\circ}$ such that $f^{\prime \prime} \in L_{1}[a, b]$, where $a, b \in I$ with $a<b$. If $\left|f^{\prime \prime}\right|$ is a convex on $[a, b]$, then the following inequality holds:

$$
\begin{aligned}
& \left|\frac{1}{6}\left[f(a)+4 f\left(\frac{a+b}{2}\right)+f(b)\right]-\frac{1}{b-a} \int_{a}^{b} f(x) d x\right| \\
\leq & \frac{(b-a)^{2}}{162}\left[\left|f^{\prime \prime}(a)\right|+\left|f^{\prime \prime}(b)\right|\right] .
\end{aligned}
$$

Theorem 3 (See [9]). Let $f: I \subset \mathbb{R} \rightarrow \mathbb{R}$ be a twice differentiable mapping on $I^{\circ}$ such that $f^{\prime \prime} \in L_{1}[a, b]$, where $a, b \in I$ with $a<b$. If $\left|f^{\prime \prime}\right|^{q}$ is a convex on $[a, b]$ and $q \geq 1$, then the following inequality holds:

$$
\begin{aligned}
& \left|\frac{1}{6}\left[f(a)+4 f\left(\frac{a+b}{2}\right)+f(b)\right]-\frac{1}{b-a} \int_{a}^{b} f(x) d x\right| \\
& \leq(b-a)^{2}\left(\frac{1}{162}\right)^{1-\frac{1}{q}}\left\{\left(\frac{59}{3^{5} 2^{7}}\left|f^{\prime \prime}(b)\right|^{q}+\frac{133}{3^{5} 2^{7}}\left|f^{\prime \prime}(a)\right|^{q}\right)^{\frac{1}{q}}\right.
\end{aligned}
$$




$$
\left.+\left(\frac{133}{3^{5} 2^{7}}\left|f^{\prime \prime}(b)\right|^{q}+\frac{59}{3^{5} 2^{7}}\left|f^{\prime \prime}(a)\right|^{q}\right)^{\frac{1}{q}}\right\}
$$

where $\frac{1}{p}+\frac{1}{q}=1$.

For recent refinements, counterparts, generalizations and new Simpson's type inequalities, see the papers $[1,4,6]$.

The main purpose of this paper is to give some generalizations of integral inequalities for convex and $m$-convex functions by using a more general lemma similar to Lemma 1. Some comparisons and applications to special means related to our results are also given.

\section{MAin Results}

Throughout this paper, we will assume that $I \subset \mathbb{R}$. To prove our main theorems we need the following lemma involving a new kernel.

Lemma 2. Let $f: I \subset \mathbb{R} \rightarrow \mathbb{R}$ be a twice differentiable mapping on $I^{\circ}$ such that $f^{\prime \prime} \in L_{1}[a, b]$, where $a, b \in I$ with $a<b$ and $r \in \mathbb{R}^{+}$then the following equality holds:

$$
\begin{aligned}
& \frac{1}{r(r+1)}[f(a)+f(b)]+\frac{2}{r+1} f\left(\frac{a+b}{2}\right)-\frac{2}{r(b-a)} \int_{a}^{b} f(x) d x \\
= & (b-a)^{2} \int_{0}^{1} k(t) f^{\prime \prime}(t b+(1-t) a) d t
\end{aligned}
$$

where

$$
k(t)=\left\{\begin{array}{ll}
\frac{t}{r}\left(\frac{1}{r+1}-t\right), & t \in\left[0, \frac{1}{2}\right) \\
(1-t)\left(\frac{t}{r}-\frac{1}{r+1}\right), & t \in\left[\frac{1}{2}, 1\right]
\end{array} .\right.
$$

Proof. By definition of $k(t)$, we can write

$$
\begin{aligned}
I= & \int_{0}^{1} k(t) f^{\prime \prime}(t b+(1-t) a) d t \\
= & \int_{0}^{\frac{1}{2}} \frac{t}{r}\left(\frac{1}{r+1}-t\right) f^{\prime \prime}(t b+(1-t) a) d t \\
& +\int_{\frac{1}{2}}^{1}(1-t)\left(\frac{t}{r}-\frac{1}{r+1}\right) f^{\prime \prime}(t b+(1-t) a) d t .
\end{aligned}
$$


Integrating the right hand side of the above equality by parts twice, we have

$$
\begin{aligned}
& \int_{0}^{\frac{1}{2}} \frac{t}{r}\left(\frac{1}{r+1}-t\right) f^{\prime \prime}(t b+(1-t) a) d t \\
= & -\frac{r-1}{4 r(r+1)(b-a)} f^{\prime}\left(\frac{a+b}{2}\right) \\
& +\frac{1}{(b-a)^{2}}\left[\frac{1}{r+1} f\left(\frac{a+b}{2}\right)+\frac{1}{r(r+1)} f(a)-\frac{2}{r} \int_{0}^{\frac{1}{2}} f(t b+(1-t) a) d t\right]
\end{aligned}
$$

and

$$
\begin{aligned}
& \int_{\frac{1}{2}}^{1}(1-t)\left(\frac{t}{r}-\frac{1}{r+1}\right) f^{\prime \prime}(t b+(1-t) a) d t \\
= & \frac{r-1}{4 r(r+1)(b-a)} f^{\prime}\left(\frac{a+b}{2}\right) \\
& +\frac{1}{(b-a)^{2}}\left[\frac{1}{r+1} f\left(\frac{a+b}{2}\right)+\frac{1}{r(r+1)} f(b)-\frac{2}{r} \int_{\frac{1}{2}}^{1} f(t b+(1-t) a) d t\right] .
\end{aligned}
$$

By addition and using the change of variable $x=t b+(1-t) a$ for $t \in[0,1]$ and multiplying the both sides by $(b-a)^{2}$, we obtain (2.1).

Remark 1. If we choose $r=2$ in (2.1), we get the equality (1.1).

Theorem 4. Let $f: I \subset \mathbb{R} \rightarrow \mathbb{R}$ be a twice differentiable mapping on $I^{\circ}$ such that $f^{\prime \prime} \in L_{1}[a, b]$, where $a, b \in I$ with $a<b$ and $r \in \mathbb{R}^{+}$. If $\left|f^{\prime \prime}\right|$ is convex, then we have the following inequality;

$$
\begin{aligned}
& \left|\frac{1}{r(r+1)}[f(a)+f(b)]+\frac{2}{r+1} f\left(\frac{a+b}{2}\right)-\frac{2}{r(b-a)} \int_{a}^{b} f(x) d x\right| \\
& \leq M(b-a)^{2}\left[\left|f^{\prime \prime}(a)\right|+\left|f^{\prime \prime}(b)\right|\right]
\end{aligned}
$$

where $M=\frac{r^{3}-3 r+6}{24 r(r+1)^{3}}$.

Proof. From Lemma 2 and by using convexity of $\left|f^{\prime \prime}(x)\right|$, we obtain

$$
\left|\frac{1}{r(r+1)}[f(a)+f(b)]+\frac{2}{r+1} f\left(\frac{a+b}{2}\right)-\frac{2}{r} \int_{0}^{1} f(t b+(1-t) a) d t\right|
$$




$$
\begin{aligned}
& \leq(b-a)^{2}\left\{\int_{0}^{\frac{1}{2}}\left|\frac{t}{r}\left(\frac{1}{r+1}-t\right)\right|\left[t\left|f^{\prime \prime}(b)\right|+(1-t)\left|f^{\prime \prime}(a)\right|\right] d t\right. \\
& \left.\quad+\int_{\frac{1}{2}}^{1}\left|(1-t)\left(\frac{t}{r}-\frac{1}{r+1}\right)\right|\left[t\left|f^{\prime \prime}(b)\right|+(1-t)\left|f^{\prime \prime}(a)\right|\right] d t\right\} \\
& =(b-a)^{2}\left\{\int_{0}^{\frac{1}{2}}\left|\frac{t}{r}\left(\frac{1}{r+1}-t\right)\right|\left[t\left|f^{\prime \prime}(b)\right|+(1-t)\left|f^{\prime \prime}(a)\right|\right] d t\right. \\
& \left.+\int_{0}^{\frac{1}{2}}\left|t\left(\frac{1-t}{r}-\frac{1}{r+1}\right)\right|\left[(1-t)\left|f^{\prime \prime}(b)\right|+t\left|f^{\prime \prime}(a)\right|\right] d t\right\} \\
& =(b-a)^{2}\left(J_{1}+J_{2}\right)
\end{aligned}
$$

where

$$
\begin{aligned}
& J_{1}=\int_{0}^{\frac{1}{2}}\left|\frac{t}{r}\left(\frac{1}{r+1}-t\right)\right|\left[t\left|f^{\prime \prime}(b)\right|+(1-t)\left|f^{\prime \prime}(a)\right| d t\right] \\
& J_{2}=\int_{\frac{1}{2}}^{1}\left|(1-t)\left(\frac{t}{r}-\frac{1}{r+1}\right)\right|\left[t\left|f^{\prime \prime}(b)\right|+(1-t)\left|f^{\prime \prime}(a)\right| d t\right] .
\end{aligned}
$$

By a little hard computation, one can see that

$$
\begin{aligned}
J_{1}= & \int_{0}^{\frac{1}{r+1}}\left|\frac{t}{r}\left(\frac{1}{r+1}-t\right)\right|\left[t\left|f^{\prime \prime}(b)\right|+(1-t)\left|f^{\prime \prime}(a)\right| d t\right] \\
& +\int_{\frac{1}{r+1}}^{\frac{1}{2}}\left|\frac{t}{r}\left(t-\frac{1}{r+1}\right)\right|\left[t\left|f^{\prime \prime}(b)\right|+(1-t)\left|f^{\prime \prime}(a)\right| d t\right] \\
= & M_{1}\left|f^{\prime \prime}(b)\right|+M_{2}\left|f^{\prime \prime}(a)\right|
\end{aligned}
$$


and

$$
\begin{aligned}
J_{2}= & \int_{\frac{1}{2}}^{\frac{r}{r+1}}\left|t\left(\frac{1-t}{r}-\frac{1}{r+1}\right)\right|\left[(1-t)\left|f^{\prime \prime}(b)\right|+t\left|f^{\prime \prime}(a)\right|\right] d t \\
& +\int_{\frac{r}{r+1}}^{1}\left|t\left(\frac{1-t}{r}-\frac{1}{r+1}\right)\right|\left[(1-t)\left|f^{\prime \prime}(b)\right|+t\left|f^{\prime \prime}(a)\right|\right] d t \\
= & M_{1}\left|f^{\prime \prime}(a)\right|+M_{2}\left|f^{\prime \prime}(b)\right|
\end{aligned}
$$

where

$$
M_{1}=\frac{3 r^{4}+4 r^{3}-6 r^{2}-12 r+27}{192 r(r+1)^{4}} \text { and } M_{2}=\frac{5 r^{4}+4 r^{3}-18 r^{2}+36 r+21}{192 r(r+1)^{4}}
$$

By taking into account $J_{1}, J_{2}, M_{1}$ and $M_{2}$ in (2.3), we obtain

$$
\begin{aligned}
J_{1}+J_{2} & =\left(M_{1}+M_{2}\right)\left[\left|f^{\prime \prime}(a)\right|+\left|f^{\prime \prime}(b)\right|\right] \\
& =M\left[\left|f^{\prime \prime}(a)\right|+\left|f^{\prime \prime}(b)\right|\right]
\end{aligned}
$$

which completes the proof.

Corollary 1. If we take $r=1$ in (2.2) we obtain the following inequality:

$$
\begin{aligned}
& \quad\left|\frac{f(a)+f(b)}{2}+f\left(\frac{a+b}{2}\right)-\frac{2}{b-a} \int_{a}^{b} f(x) d x\right| \\
& \leq \frac{(b-a)^{2}}{48}\left[\left|f^{\prime \prime}(a)\right|+\left|f^{\prime \prime}(b)\right|\right] .
\end{aligned}
$$

Corollary 2. The following table shows the results in (2.2) for several values of $r$. In the table the left hand side of (2.2) is given by LHS and the right one is given by RHS. 


\begin{tabular}{|l|l|l|}
\hline$r$ & $L H S$ & $R H S$ \\
\hline 1 & $\frac{f(a)+f(b)}{2}+f\left(\frac{a+b}{2}\right)-\frac{2}{(b-a)} \int_{a}^{b} f(x) d x$ & $\frac{(b-a)^{2}}{48}\left[\left|f^{\prime \prime}(a)\right|+\left|f^{\prime \prime}(b)\right|\right]$ \\
\hline 2 & $\frac{1}{6}\left[f(a)+4 f\left(\frac{a+b}{2}\right)+f(b)\right]-\frac{1}{(b-a)} \int_{a}^{b} f(x) d x$ & $\frac{(b-a)^{2}}{162}\left[\left|f^{\prime \prime}(a)\right|+\left|f^{\prime \prime}(b)\right|\right]$ \\
\hline 3 & $\frac{1}{12}\left[f(a)+6 f\left(\frac{a+b}{2}\right)+f(b)\right]-\frac{2}{3(b-a)} \int_{a}^{b} f(x) d x$ & $\frac{(b-a)^{2}}{192}\left[\left|f^{\prime \prime}(a)\right|+\left|f^{\prime \prime}(b)\right|\right]$ \\
\hline 24 & $\frac{1}{600}\left[f(a)+48 f\left(\frac{a+b}{2}\right)+f(b)\right]-\frac{1}{12(b-a)} \int_{a}^{b} f(x) d x$ & $\frac{(b-a)^{2}}{654}\left[\left|f^{\prime \prime}(a)\right|+\left|f^{\prime \prime}(b)\right|\right]$ \\
\hline 30 & $\frac{1}{930}\left[f(a)+30 f\left(\frac{a+b}{2}\right)+f(b)\right]-\frac{1}{15(b-a)} \int_{a}^{b} f(x) d x$ & $\frac{(b-a)^{2}}{797}\left[\left|f^{\prime \prime}(a)\right|+\left|f^{\prime \prime}(b)\right|\right]$ \\
\hline
\end{tabular}

From the table one can see that if we take $r \rightarrow \infty$ we obtain smaller upper bounds for the inequality (2.2).

Remark 2. If we take $r=2$ in (2.2) we obtain (1.2).

Example 1. Under the assumptions of Theorem 4, if we choose $[a, b]=[0,1]$ then we can give the following graphics for some special cases of $f$ by Mathematica programme. In these graphics $x$-axis shows the values of $r \in \mathbb{R}^{+}$:

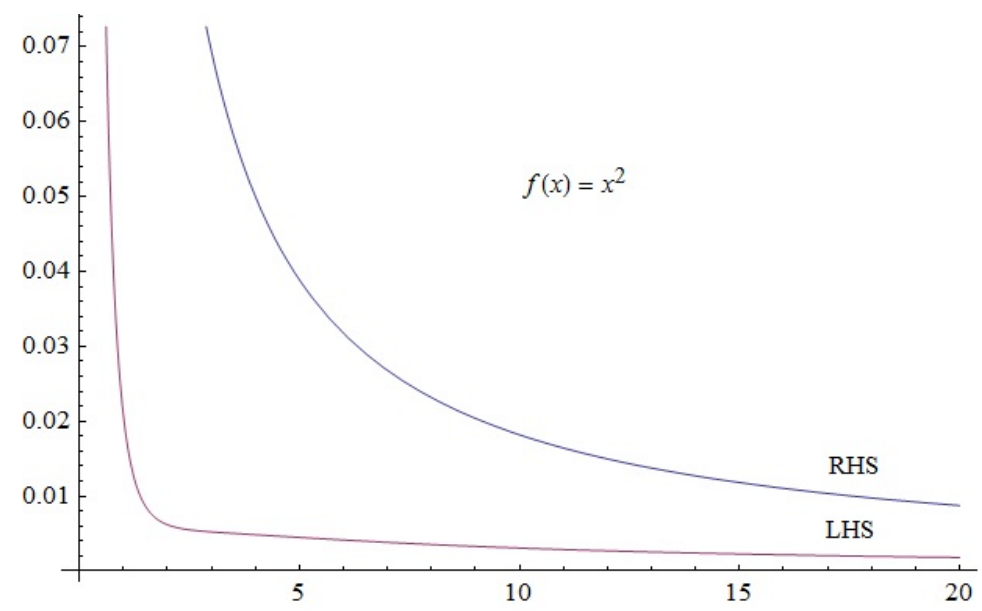




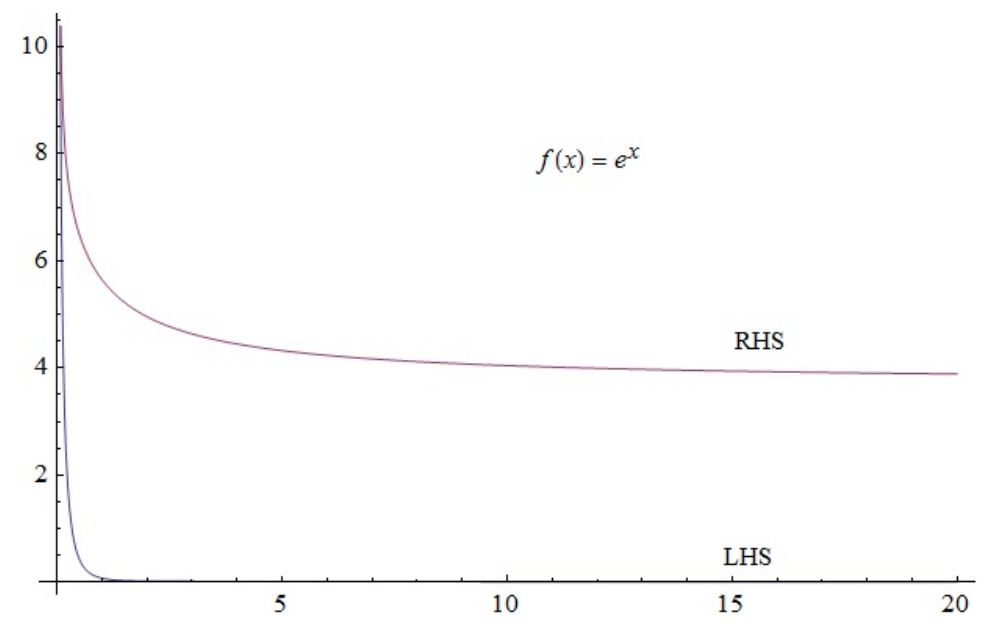

Theorem 5. Let $f: I \subset \mathbb{R} \rightarrow \mathbb{R}$ be a twice differentiable mapping on $I^{\circ}$ such that $f^{\prime \prime} \in L_{1}[a, b]$ and if $\left|f^{\prime \prime}\right|^{q}$ is convex on $[a, b]$ where $a, b \in I$ with $a<b, r \in \mathbb{R}^{+}$ and $q \geq 1$, then the following inequality holds:

$$
\begin{aligned}
& \left|\frac{1}{r(r+1)}[f(a)+f(b)]+\frac{2}{r+1} f\left(\frac{a+b}{2}\right)-\frac{2}{r(b-a)} \int_{a}^{b} f(x) d x\right| \\
& \leq(b-a)^{2}\left(\frac{r^{3}-3 r+6}{24 r(r+1)^{3}}\right)^{1-\frac{1}{q}}\left\{\left[\left(M_{1}\left|f^{\prime \prime}(b)\right|^{q}+M_{2}\left|f^{\prime \prime}(a)\right|^{q}\right)^{\frac{1}{q}}\right]\right. \\
& \left.\quad+\left[\left(M_{2}\left|f^{\prime \prime}(b)\right|^{q}+M_{1}\left|f^{\prime \prime}(a)\right|^{q}\right)^{\frac{1}{q}}\right]\right\}
\end{aligned}
$$

where $M_{1}=\frac{3 r^{4}+4 r^{3}-6 r^{2}-12 r+27}{192 r(r+1)^{4}}$ and $M_{2}=\frac{5 r^{4}+4 r^{3}-18 r^{2}+36 r+21}{192 r(r+1)^{4}}$.

Proof. From Lemma 2 and by using the well known power-mean inequality for $q \geq 1$, we have

$$
\begin{aligned}
& \left|\frac{1}{r(r+1)}[f(a)+f(b)]+\frac{2}{r+1} f\left(\frac{a+b}{2}\right)-\frac{2}{r(b-a)} \int_{a}^{b} f(x) d x\right| \\
& \leq(b-a)^{2} \int_{0}^{1}|k(t)|\left|f^{\prime \prime}(t b+(1-t) a)\right| d t \\
& \leq(b-a)^{2}\left(\int_{0}^{\frac{1}{2}}\left|\frac{t}{r}\left(\frac{1}{r+1}-t\right)\right| d t\right)^{1-\frac{1}{q}}
\end{aligned}
$$


GENERALIZATIONS OF INTEGRAL INEQUALITIES

449

$$
\begin{aligned}
& \cdot\left(\int_{0}^{\frac{1}{2}}\left|\frac{t}{r}\left(\frac{1}{r+1}-t\right)\right|\left|f^{\prime \prime}(t b+(1-t) a)\right|^{q} d t\right)^{\frac{1}{q}} \\
& +\left(\int_{\frac{1}{2}}^{1}\left|(1-t)\left(\frac{t}{r}-\frac{1}{r+1}\right)\right| d t\right)^{1-\frac{1}{q}} \\
& \cdot\left(\int_{\frac{1}{2}}^{1}\left|(1-t)\left(\frac{t}{r}-\frac{1}{r+1}\right)\right|\left|f^{\prime \prime}(t b+(1-t) a)\right|^{q} d t\right)^{\frac{1}{q}} .
\end{aligned}
$$

On the other hand, since $\left|f^{\prime \prime}\right|^{q}$ is convex on $[a, b]$, we have

$$
\begin{aligned}
& \int_{0}^{\frac{1}{2}}\left|\frac{t}{r}\left(\frac{1}{r+1}-t\right)\right|\left|f^{\prime \prime}(t b+(1-t) a)\right|^{q} d t \\
\leq & \int_{0}^{\frac{1}{2}}\left|\frac{t}{r}\left(\frac{1}{r+1}-t\right)\right|\left[t\left|f^{\prime \prime}(b)\right|^{q}+(1-t)\left|f^{\prime \prime}(a)\right|^{q}\right] d t \\
= & \int_{0}^{\frac{1}{r+1}}\left|\frac{t}{r}\left(\frac{1}{r+1}-t\right)\right|\left[t\left|f^{\prime \prime}(b)\right|^{q}+(1-t)\left|f^{\prime \prime}(a)\right|^{q}\right] d t \\
& +\int_{\frac{1}{r+1}}^{\frac{1}{2}}\left|\frac{t}{r}\left(t-\frac{1}{r+1}\right)\right|\left[t\left|f^{\prime \prime}(b)\right|^{q}+(1-t)\left|f^{\prime \prime}(a)\right|^{q}\right] d t \\
= & M_{1}\left|f^{\prime \prime}(b)\right|^{q}+M_{2}\left|f^{\prime \prime}(a)\right|^{q}
\end{aligned}
$$

and

$$
\begin{aligned}
& \int_{\frac{1}{2}}^{1}\left|(1-t)\left(\frac{t}{r}-\frac{1}{r+1}\right)\right|\left|f^{\prime \prime}(t b+(1-t) a)\right|^{q} d t \\
\leq & \int_{\frac{1}{2}}^{1}\left|(1-t)\left(\frac{t}{r}-\frac{1}{r+1}\right)\right|\left[t\left|f^{\prime \prime}(b)\right|^{q}+(1-t)\left|f^{\prime \prime}(a)\right|^{q}\right] d t
\end{aligned}
$$




$$
\begin{aligned}
= & \int_{\frac{1}{2}}^{\frac{r}{r+1}}\left|(1-t)\left(\frac{t}{r}-\frac{1}{r+1}\right)\right|\left[t\left|f^{\prime \prime}(b)\right|^{q}+(1-t)\left|f^{\prime \prime}(a)\right|^{q}\right] d t \\
& +\int_{\frac{r}{r+1}}^{1}\left|(1-t)\left(\frac{1}{r+1}-\frac{t}{r}\right)\right|\left[t\left|f^{\prime \prime}(b)\right|^{q}+(1-t)\left|f^{\prime \prime}(a)\right|^{q}\right] d t \\
= & M_{2}\left|f^{\prime \prime}(b)\right|^{q}+M_{1}\left|f^{\prime \prime}(a)\right|^{q}
\end{aligned}
$$

where $M_{1}=\frac{3 r^{4}+4 r^{3}-6 r^{2}-12 r+27}{192 r(r+1)^{4}}$ and $M_{2}=\frac{5 r^{4}+4 r^{3}-18 r^{2}+36 r+21}{192 r(r+1)^{4}}$.

From (2.5) and (2.6) and by using the fact that

$$
\int_{0}^{\frac{1}{2}}\left|\frac{t}{r}\left(\frac{1}{r+1}-t\right)\right| d t=\int_{\frac{1}{2}}^{1}\left|(1-t)\left(\frac{t}{r}-\frac{1}{r+1}\right)\right| d t=\frac{r^{3}-3 r+6}{24 r(r+1)^{3}}
$$

we deduce

$$
\begin{aligned}
& \left|\frac{1}{r(r+1)}[f(a)+f(b)]+\frac{2}{r+1} f\left(\frac{a+b}{2}\right)-\frac{2}{r(b-a)} \int_{a}^{b} f(x) d x\right| \\
& \leq(b-a)^{2}\left(\frac{r^{3}-3 r+6}{24 r(r+1)^{3}}\right)^{1-\frac{1}{q}}\left\{\left[\left(M_{1}\left|f^{\prime \prime}(b)\right|^{q}+M_{2}\left|f^{\prime \prime}(a)\right|^{q}\right)^{\frac{1}{q}}\right]\right. \\
& \left.\quad+\left[\left(M_{2}\left|f^{\prime \prime}(b)\right|^{q}+M_{1}\left|f^{\prime \prime}(a)\right|^{q}\right)^{\frac{1}{q}}\right]\right\},
\end{aligned}
$$

which completes the proof.

Corollary 3. i) Under the assumptions of Theorem 5, if we choose $r=1$ we get the following inequality:

$$
\begin{aligned}
& \left|\frac{f(a)+f(b)}{2}+f\left(\frac{a+b}{2}\right)-\frac{2}{b-a} \int_{a}^{b} f(x) d x\right| \\
& \leq(b-a)^{2}\left(\frac{1}{48}\right)^{1-\frac{1}{q}}\left\{\left[\left(\frac{1}{192}\left|f^{\prime \prime}(b)\right|^{q}+\frac{3}{192}\left|f^{\prime \prime}(a)\right|^{q}\right)^{\frac{1}{q}}\right]\right. \\
& \left.+\left[\left(\frac{3}{192}\left|f^{\prime \prime}(b)\right|^{q}+\frac{1}{192}\left|f^{\prime \prime}(a)\right|^{q}\right)^{\frac{1}{q}}\right]\right\}
\end{aligned}
$$


ii) Let $a_{1}=\frac{1}{192}\left|f^{\prime \prime}(b)\right|^{q}, b_{1}=\frac{3}{192}\left|f^{\prime \prime}(a)\right|^{q}, a_{2}=\frac{3}{192}\left|f^{\prime \prime}(b)\right|^{q}, b_{2}=\frac{1}{192}\left|f^{\prime \prime}(a)\right|^{q}$. Here $0<\frac{1}{q}<1$, for $q>1$, using the fact that

$$
\sum_{k=1}^{n}\left(a_{k}+b_{k}\right)^{s} \leq \sum_{k=1}^{n}\left(a_{k}\right)^{s}+\sum_{k=1}^{n}\left(b_{k}\right)^{s}
$$

for $0 \leq s \leq 1, a_{1}, \ldots, a_{n} \geq 0, b_{1}, \ldots b_{n} \geq 0$, we get the following inequality:

$$
\begin{aligned}
& \left|\frac{f(a)+f(b)}{2}+f\left(\frac{a+b}{2}\right)-\frac{2}{b-a} \int_{a}^{b} f(x) d x\right| \\
\leq & (b-a)^{2}\left(\frac{1}{48}\right)^{1-\frac{1}{q}}\left\{\frac{1}{192^{1 / q}}\left|f^{\prime \prime}(b)\right|+\left(\frac{3}{192}\right)^{1 / q}\left|f^{\prime \prime}(a)\right|\right\} .
\end{aligned}
$$

Now for $q \rightarrow \infty$, we get

$$
\left|\frac{f(a)+f(b)}{2}+f\left(\frac{a+b}{2}\right)-\frac{2}{b-a} \int_{a}^{b} f(x) d x\right| \leq \frac{(b-a)^{2}}{24}\left(\left|f^{\prime \prime}(a)\right|+\left|f^{\prime \prime}(b)\right|\right) \text {. }
$$

Theorem 6. Let $f: I \subset \mathbb{R} \rightarrow \mathbb{R}$ be a twice differentiable mapping on $I^{\circ}$ such that $f^{\prime \prime} \in L_{1}[a, b]$ and $\left|f^{\prime \prime}\right|$ is $m$-convex function with $m \in(0,1]$, where $a, b \in I$ with $a<b$ and $r \in \mathbb{R}^{+}$

$$
\begin{aligned}
& \left|\frac{1}{r(r+1)}[f(a)+f(b)]+\frac{2}{r+1} f\left(\frac{a+b}{2}\right)-\frac{2}{r(b-a)} \int_{a}^{b} f(x) d x\right| \\
& \leq(b-a)^{2} M\left[m\left|f^{\prime \prime}\left(\frac{a}{m}\right)\right|+\left|f^{\prime \prime}(b)\right|\right]
\end{aligned}
$$

where $M=\frac{r^{3}-3 r+6}{24 r(r+1)^{3}}$.

Proof. From Lemma 2 and using the property of absolute value, we can write

$$
\begin{aligned}
& \left|\frac{1}{r(r+1)}[f(a)+f(b)]+\frac{2}{r+1} f\left(\frac{a+b}{2}\right)-\frac{2}{r(b-a)} \int_{a}^{b} f(x) d x\right| \\
& \leq(b-a)^{2} \int_{0}^{1}\left|k(t) f^{\prime \prime}(t b+(1-t) a)\right| d t .
\end{aligned}
$$

Since $\left|f^{\prime \prime}\right|$ is $m$-convex on $[a, b]$, we know that for any $t \in[0,1]$

$$
\left|f^{\prime \prime}(t b+(1-t) a)\right|=\left|f^{\prime \prime}\left(t b+m(1-t) \frac{a}{m}\right)\right|
$$


hence it follows that

$$
\leq t\left|f^{\prime \prime}(b)\right|+m(1-t)\left|f^{\prime \prime}\left(\frac{a}{m}\right)\right|
$$

$$
\begin{aligned}
& (b-a)^{2} \int_{0}^{1}|k(t)|\left[t\left|f^{\prime \prime}(b)\right|+m(1-t)\left|f^{\prime \prime}\left(\frac{a}{m}\right)\right|\right] d t \\
= & (b-a)^{2}\left\{\int_{0}^{\frac{1}{2}}\left|\frac{t}{r}\left(\frac{1}{r+1}-t\right)\right|\left[t\left|f^{\prime \prime}(b)\right|+m(1-t)\left|f^{\prime \prime}\left(\frac{a}{m}\right)\right|\right] d t\right. \\
& \left.+\int_{\frac{1}{2}}^{1}\left|(1-t)\left(\frac{t}{r}-\frac{1}{r+1}\right)\right|\left[t\left|f^{\prime \prime}(b)\right|+m(1-t)\left|f^{\prime \prime}\left(\frac{a}{m}\right)\right|\right] d t\right\} \\
= & J_{1}+J_{2}
\end{aligned}
$$

where

$$
\begin{aligned}
& J_{1}=\int_{0}^{\frac{1}{2}}\left|\frac{t}{r}\left(\frac{1}{r+1}-t\right)\right|\left[t\left|f^{\prime \prime}(b)\right|+m(1-t)\left|f^{\prime \prime}\left(\frac{a}{m}\right)\right|\right] d t \\
& J_{2}=\int_{\frac{1}{2}}^{1}\left|(1-t)\left(\frac{t}{r}-\frac{1}{r+1}\right)\right|\left[t\left|f^{\prime \prime}(b)\right|+m(1-t)\left|f^{\prime \prime}\left(\frac{a}{m}\right)\right|\right] d t .
\end{aligned}
$$

If we compute $J_{1}$ and $J_{2}$, we have

$$
\begin{aligned}
J_{1}= & \int_{0}^{\frac{1}{r+1}}\left|\frac{t}{r}\left(\frac{1}{r+1}-t\right)\right|\left[t\left|f^{\prime \prime}(b)\right|+m(1-t)\left|f^{\prime \prime}\left(\frac{a}{m}\right)\right|\right] d t \\
& +\int_{\frac{1}{r+1}}^{\frac{1}{2}}\left|\frac{t}{r}\left(t-\frac{1}{r+1}\right)\right|\left[t\left|f^{\prime \prime}(b)\right|+m(1-t)\left|f^{\prime \prime}\left(\frac{a}{m}\right)\right|\right] d t \\
= & M_{1}\left|f^{\prime \prime}(b)\right|+M_{2} m\left|f^{\prime \prime}\left(\frac{a}{m}\right)\right|
\end{aligned}
$$

and

$$
J_{2}=\int_{\frac{1}{2}}^{\frac{r}{r+1}}\left|(1-t)\left(\frac{1}{r+1}-\frac{t}{r}\right)\right|\left[t\left|f^{\prime \prime}(b)\right|+m(1-t)\left|f^{\prime \prime}\left(\frac{a}{m}\right)\right|\right] d t
$$




$$
\begin{aligned}
& +\int_{\frac{r}{r+1}}^{1}\left|(1-t)\left(\frac{t}{r}-\frac{1}{r+1}\right)\right|\left[t\left|f^{\prime \prime}(b)\right|+m(1-t)\left|f^{\prime \prime}\left(\frac{a}{m}\right)\right|\right] d t \\
= & M_{1} m\left|f^{\prime \prime}\left(\frac{a}{m}\right)\right|+M_{2}\left|f^{\prime \prime}(b)\right|
\end{aligned}
$$

where $M_{1}=\frac{3 r^{4}+4 r^{3}-6 r^{2}-12 r+27}{192 r(r+1)^{4}}$ and $M_{2}=\frac{5 r^{4}+4 r^{3}-18 r^{2}+36 r+21}{192 r(r+1)^{4}}$.

By taking into account $J_{1}$ and $J_{2}$, we obtain the desired result.

Theorem 7. Let $f: I \subset \mathbb{R} \rightarrow \mathbb{R}$ be a twice differentiable mapping on $I^{\circ}$ such that $f^{\prime \prime} \in L_{1}[a, b]$ and if $\left|f^{\prime \prime}\right|^{q}$ is $m$-convex on $[a, b]$ and $m \in(0,1]$ where $a, b \in I$ with $a<b, r \in \mathbb{R}^{+}$and $q \geq 1$, then the following inequality holds;

$$
\begin{aligned}
& \left|\frac{1}{r(r+1)}[f(a)+f(b)]+\frac{2}{r+1} f\left(\frac{a+b}{2}\right)-\frac{2}{r(b-a)} \int_{a}^{b} f(x) d x\right| \\
& \leq(b-a)^{2}\left(\frac{r^{3}-3 r+6}{24 r(r+1)^{3}}\right)^{1-\frac{1}{q}}\left\{\left\{\left(M_{1}\left|f^{\prime \prime}(b)\right|^{q}+M_{2} m\left|f^{\prime \prime}\left(\frac{a}{m}\right)\right|^{q}\right)^{\frac{1}{q}}\right\}\right. \\
& \left.\quad+\left(M_{2}\left|f^{\prime \prime}(b)\right|^{q}+M_{1} m\left|f^{\prime \prime}\left(\frac{a}{m}\right)\right|^{q}\right)^{\frac{1}{q}}\right\}
\end{aligned}
$$

where $M_{1}=\frac{3 r^{4}+4 r^{3}-6 r^{2}-12 r+27}{192 r(r+1)^{4}}$ and $M_{2}=\frac{5 r^{4}+4 r^{3}-18 r^{2}+36 r+21}{192 r(r+1)^{4}}$.

Proof. From Lemma 2 and by using the well known power-mean inequality, we have

$$
\begin{aligned}
& \left|\frac{1}{r(r+1)}[f(a)+f(b)]+\frac{2}{r+1} f\left(\frac{a+b}{2}\right)-\frac{2}{r(b-a)} \int_{a}^{b} f(x) d x\right| \\
\leq & (b-a)^{2} \int_{0}^{1}|k(t)|\left|f^{\prime \prime}(t b+(1-t) a)\right| d t \\
\leq & (b-a)^{2}\left(\int_{0}^{\frac{1}{2}}\left|\frac{t}{r}\left(\frac{1}{r+1}-t\right)\right| d t\right)^{1-\frac{1}{q}} \\
& \cdot\left(\int_{0}^{\frac{1}{2}}\left|\frac{t}{r}\left(\frac{1}{r+1}-t\right)\right|\left|f^{\prime \prime}(t b+(1-t) a)\right|^{q} d t\right)^{\frac{1}{q}}
\end{aligned}
$$


454

M. E. ÖZDEMIR, A. EKINCI, AND A. O. AKDEMIR

$$
\begin{aligned}
& +\left(\int_{\frac{1}{2}}^{1}\left|(1-t)\left(\frac{t}{r}-\frac{1}{r+1}\right)\right| d t\right)^{1-\frac{1}{q}} \\
& \cdot\left(\int_{\frac{1}{2}}^{1}\left|(1-t)\left(\frac{t}{r}-\frac{1}{r+1}\right)\right|\left|f^{\prime \prime}(t b+(1-t) a)\right|^{q} d t\right)^{\frac{1}{q}} .
\end{aligned}
$$

Since $\left|f^{\prime \prime}\right|^{q}$ is $m$-convex, we have

$$
\begin{aligned}
& \int_{0}^{\frac{1}{2}}\left|\frac{t}{r}\left(\frac{1}{r+1}-t\right)\right|\left|f^{\prime \prime}(t b+(1-t) a)\right|^{q} d t \\
\leq & \int_{0}^{\frac{1}{2}}\left|\frac{t}{r}\left(\frac{1}{r+1}-t\right)\right|\left[t\left|f^{\prime \prime}(b)\right|^{q}+m(1-t)\left|f^{\prime \prime}\left(\frac{a}{m}\right)\right|^{q}\right] d t \\
= & \int_{0}^{\frac{1}{r+1}}\left|\frac{t}{r}\left(\frac{1}{r+1}-t\right)\right|\left[t\left|f^{\prime \prime}(b)\right|^{q}+m(1-t)\left|f^{\prime \prime}\left(\frac{a}{m}\right)\right|^{q}\right] d t \\
& +\int_{\frac{1}{r+1}}^{\frac{1}{2}}\left|\frac{t}{r}\left(t-\frac{1}{r+1}\right)\right|\left[t\left|f^{\prime \prime}(b)\right|^{q}+m(1-t)\left|f^{\prime \prime}\left(\frac{a}{m}\right)\right|^{q}\right] d t \\
= & M_{1}\left|f^{\prime \prime}(b)\right|^{q}+M_{2} m\left|f^{\prime \prime}\left(\frac{a}{m}\right)\right|^{q}
\end{aligned}
$$

and

$$
\begin{aligned}
& \int_{\frac{1}{2}}^{1}\left|(1-t)\left(\frac{t}{r}-\frac{1}{r+1}\right)\right|\left|f^{\prime \prime}(t b+(1-t) a)\right|^{q} d t \\
\leq & \int_{\frac{1}{2}}^{1}\left|(1-t)\left(\frac{t}{r}-\frac{1}{r+1}\right)\right|\left[t\left|f^{\prime \prime}(b)\right|^{q}+m(1-t)\left|f^{\prime \prime}\left(\frac{a}{m}\right)\right|^{q}\right] d t \\
= & \int_{\frac{1}{2}}^{\frac{r}{r+1}}\left|(1-t)\left(\frac{t}{r}-\frac{1}{r+1}\right)\right|\left[t\left|f^{\prime \prime}(b)\right|^{q}+m(1-t)\left|f^{\prime \prime}\left(\frac{a}{m}\right)\right|^{q}\right] d t
\end{aligned}
$$




$$
\begin{aligned}
& +\int_{\frac{r}{r+1}}^{1}\left|(1-t)\left(\frac{1}{r+1}-\frac{t}{r}\right)\right|\left[t\left|f^{\prime \prime}(b)\right|^{q}+m(1-t)\left|f^{\prime \prime}\left(\frac{a}{m}\right)\right|^{q}\right] d t \\
= & M_{2}\left|f^{\prime \prime}(b)\right|^{q}+M_{1} m\left|f^{\prime \prime}\left(\frac{a}{m}\right)\right|^{q}
\end{aligned}
$$

where $M_{1}=\frac{3 r^{4}+4 r^{3}-6 r^{2}-12 r+27}{192 r(r+1)^{4}}$ and $M_{2}=\frac{5 r^{4}+4 r^{3}-18 r^{2}+36 r+21}{192 r(r+1)^{4}}$.

From (2.9) and (2.10) and by using the fact that

$$
\int_{0}^{\frac{1}{2}}\left|\frac{t}{r}\left(\frac{1}{r+1}-t\right)\right| d t=\int_{\frac{1}{2}}^{1}\left|(1-t)\left(\frac{t}{r}-\frac{1}{r+1}\right)\right| d t=\frac{r^{3}-3 r+6}{24 r(r+1)^{3}}
$$

we obtain

$$
\begin{aligned}
& \left|\frac{1}{r(r+1)}[f(a)+f(b)]+\frac{2}{r+1} f\left(\frac{a+b}{2}\right)-\frac{2}{r(b-a)} \int_{a}^{b} f(x) d x\right| \\
& \leq(b-a)^{2}\left(\frac{r^{3}-3 r+6}{24 r(r+1)^{3}}\right)^{1-\frac{1}{q}}\left\{\left(M_{1}\left|f^{\prime \prime}(b)\right|^{q}+M_{2} m\left|f^{\prime \prime}\left(\frac{a}{m}\right)\right|^{q}\right)^{\frac{1}{q}}\right. \\
& \left.\quad+\left(M_{2}\left|f^{\prime \prime}(b)\right|^{q}+M_{1} m\left|f^{\prime \prime}\left(\frac{a}{m}\right)\right|^{q}\right)^{\frac{1}{q}}\right\},
\end{aligned}
$$

which completes the proof.

\section{Applications to SPeCial MEANS}

Now, we consider some applications of our theorems to the following special means.

a) The arithmetic mean:

$$
A=A(a, b):=\frac{a+b}{2}, a, b \geq 0,
$$

b) The harmonic mean:

$$
H=H(a, b):=\frac{2 a b}{a+b}, a, b \geq 0,
$$

c) The logarithmic mean:

$$
L=L(a, b):=\left\{\begin{array}{ll}
a & \text { if } a=b \\
\frac{b-a}{\ln b-\ln a} & \text { if } a \neq b
\end{array}, a, b \geq 0,\right.
$$

d) The $p$-logarithmic mean: 


$$
L_{p}=L_{p}(a, b):=\left\{\begin{array}{ll}
{\left[\frac{b^{p+1}-a^{p+1}}{(p+1)(b-a)}\right]^{1 / p}} & \text { if } a \neq b \\
a & \text { if } a=b
\end{array}, p \in \mathbb{R} \backslash\{-1,0\} ; a, b>0 .\right.
$$

Proposition 1. Let $a, b, n \in \mathbb{R}$, then we have

$$
\left|A\left(a^{n}, b^{n}\right)+A^{n}(a, b)-2 L_{n}^{n}(a, b)\right| \leq \frac{(b-a)^{2}}{24} n(n-1) A\left(|a|^{n-2},|b|^{n-2}\right)
$$

and

$$
\left|\frac{1}{3} A\left(a^{n}, b^{n}\right)+\frac{2}{3} A^{n}(a, b)-L_{n}^{n}(a, b)\right| \leq \frac{(b-a)^{2}}{81} n(n-1) A\left(|a|^{n-2},|b|^{n-2}\right)
$$

Proof. The assertion follows from Theorem 4 applied for $f(x)=x^{n}, x \in[a, b]$ with $r=1$ for the first inequality and $r=2$ for the second inequality.

And especially for $f(x)=\frac{1}{x}$, we can get

$$
\left|H(a, b)+A^{-1}(a, b)-2 L(a, b)\right| \leq \frac{(b-a)^{2}}{12} A\left(|a|^{-3},|b|^{-3}\right)
$$

for $r=1$ and

$$
\left|\frac{1}{3} H(a, b)+\frac{2}{3} A^{n}(a, b)-L(a, b)\right| \leq \frac{(b-a)^{2}}{81} 2 A\left(|a|^{-3},|b|^{-3}\right)
$$

for $r=2$.

Proposition 2. Let $a, b, n \in \mathbb{R}$, then we have

$$
\left|A\left(a^{n}, b^{n}\right)+A^{n}(a, b)-2 L_{n}^{n}(a, b)\right| \leq \frac{(b-a)^{2}}{24} n(n-1) A\left(m|a|^{n-2},|b|^{n-2}\right)
$$

and

$$
\left|\frac{1}{3} A\left(a^{n}, b^{n}\right)+\frac{2}{3} A^{n}(a, b)-L_{n}^{n}(a, b)\right| \leq \frac{(b-a)^{2}}{216} n(n-1) A\left(m|a|^{n-2},|b|^{n-2}\right)
$$

Proof. The assertion follows from Theorem 6 applied for $f(x)=x^{n}, x \in[a, b]$ with $r=1$ for the first inequality and $r=2$ for the second inequality.

\section{REFERENCES}

[1] M. Alomari, M. Darus, and S. S. Dragomir, "New inequalities of Simpson's type for s-convex functions with applications," RGMIA Res. Rep. Coll., vol. 12, no. 4, p. Article 9, 2009.

[2] M. K. Bakula, M. E. Özdemir, and J. E. Pecaric, "Hadamard type inequalities for $m$-convex and $(\alpha, m)$-convex functions," JIPAM, J. Inequal. Pure Appl. Math., vol. 9, no. 4, pp. 12, Paper No. 96, 2008.

[3] M. K. Bakula, J. E. Pecaric, and M. Ribičić, "Companion inequalities to Jensen's inequality for $m$-convex and $(\alpha, m)$-convex functions," JIPAM, J. Inequal. Pure Appl. Math., vol. 7, no. 5, pp. 15, Paper No. 194, 2006. 
[4] S. S. Dragomir, R. P. Agarwal, and P. Cerone, "On Simpson's inequality and applications," J. Inequal. Appl., vol. 5, no. 6, pp. 533-579, 2000.

[5] S. S. Dragomir and G. Toader, "Some inequalities for $m$-convex functions," Stud. Univ. BabeşBolyai, Math., vol. 38, no. 1, pp. 21-28, 1993.

[6] Z. Liu, "An inequality of Simpson type," Proc. R. Soc. Lond., Ser. A, Math. Phys. Eng. Sci., vol. 461, no. 2059, pp. 2155-2158, 2005.

[7] M. E. Özdemir, E. Set, and M. Z. Sarı kaya, "Some new Hadamard's type inequalities for coordinated $m$-convex and $(\alpha, m)$-convex functions," Hacettepe J. of. Math. and Ist., vol. 40, pp. 219-229, 2011.

[8] M. E. Özdemir, M. Avc1, and E. Set, "On some inequalities of Hermite-Hadamard type via $m$ convexity," Appl. Math. Lett., vol. 23, no. 9, pp. 1065-1070, 2010.

[9] M. Z. Sarı kaya, E. Set, and M. E. Özdemir, "On new inequalities of Simpson's type for functions whose second derivatives absolute values are convex," RGMIA Res. Rep. Coll., vol. 13, no. 1, p. Article 1, 2010.

[10] G. Toader, "On a generalization of the convexity," Math., Rev. Anal. Numér. Théor. Approximation, Math., vol. 30(53), no. 1, pp. 83-87, 1988.

\section{Authors' addresses}

M. Emin Özdemir

Atatürk University, K.K. Education Faculty, Department of Mathematics, 25240, ERZURUM TURKEY

E-mail address: emoseatauni.edu.tr

Alper Ekinci

Ağrı İbrahim Çeçen University, Faculty of Science and Letters, Department of Mathematics, 04100 AĞRI TURKEY

E-mail address: alperekinci@hotmail.com

Ahmet Ocak Akdemir

A ğrı İbrahim Çeçen University, Faculty of Science and Letters, Department of Mathematics, 04100 AĞRI TURKEY

E-mail address: ahmetakdemir@agri.edu.tr 